\title{
École élémentaire et enseignement des langues
}

Jean Duverger

\section{(2) OpenEdition}

Journals

Édition électronique

URL : http://journals.openedition.org/trema/259

DOI : 10.4000/trema.259

ISSN : 2107-0997

\section{Éditeur}

Faculté d'Éducation de l'université de Montpellier

\section{Édition imprimée}

Date de publication : 1 septembre 2007

Pagination : 17-22

ISSN : 1167-315X

\section{Référence électronique}

Jean Duverger, «École élémentaire et enseignement des langues », Tréma [En ligne], 28 | 2007, mis en ligne le 13 janvier 2010, consulté le 01 mai 2019. URL : http://journals.openedition.org/trema/259 DOI : 10.4000/trema.259

Ce document a été généré automatiquement le 1 mai 2019.

Trema 


\title{
École élémentaire et enseignement des langues
}

\author{
Jean Duverger
}

1 L'histoire de l'enseignement des langues étrangères à l'école primaire est relativement courte et récente, mais mon propos n'est pas ici de faire un historique de la question. Simplement disons que le démarrage institutionnel quelque peu significatif date d'un peu plus d'une quinzaine d'années, avec l'EPLV (expérimentation contrôlée de l'enseignement d'une langue vivante étrangère à l'école primaire) et qu'au cours de cette courte période, les péripéties de toutes natures n'ont pas manqué, dues tout à la fois à des résistances profondes de type sociopolitique et culturel, (traditions de jacobinisme, de nationalisme monolingue, de centralisme du ministère de l'éducation)

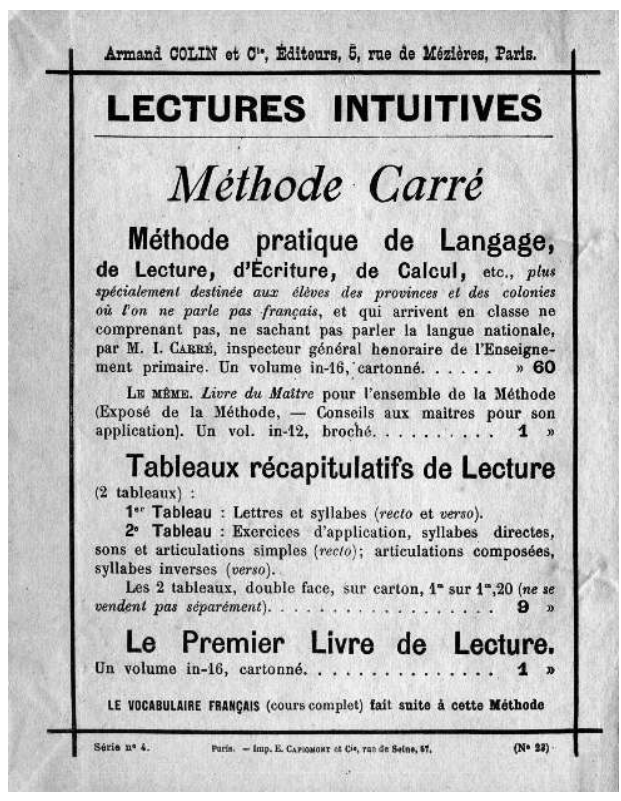
et à des carences institutionnelles notables : absence d'objectifs clairs, (on est allé de l'initiation au pré - apprentissage (?) en passant par la sensibilisation ) et impréparation totale au niveau de la formation des maitres.

2 Notons que parallèlement à ces balbutiements en matière de langues étrangères à l'école élémentaire, l'enseignement des langues régionales se développait également, mais avec les mêmes difficultés, et en partie pour les mêmes raisons. 


\section{Alors où en est-on en France en 2006 ? Comment peut-on problématiser la situation ?}

On observera d'abord que même si ce démarrage de l'enseignement des langues autres que le français à l'école élémentaire est long et balbutiant, il semble bien que le phénomène soit irréversible ; petit à petit, dans ce contexte de mondialisation, d'accélération des échanges et de multiplication des communications, les mentalités évoluent et le monolinguisme apparaît de plus en plus comme un sérieux handicap.

4 Pour la première fois dans l'histoire institutionnelle de l'école élémentaire, les textes officiels de 2002 publiés par le ministère de l'éducation nationale formulent des programmes et instructions en matière de langues étrangères et régionales.

5 De plus et c'est nouveau, ces textes s'appliquent pour tout le monde. Il y a obligation. On n'est donc plus dans la logique du volontariat, des classes expérimentales ou d'un élitisme explicite ou implicite : le temps - pas si lointain - où de savants docteurs affirmaient sans rire qu'à l'école élémentaire, l'introduction même légère d'une autre langue que la langue maternelle pouvait perturber le développement du langage, empêcher d'apprendre à lire ou provoquer des dyslexies et autres dysfonctionnements mentaux semble un peu révolu, au moins provisoirement (les rechutes sont sans doute néanmoins possibles).

Mais se posent cependant encore deux questions fondamentales dont les réponses ne semblent pas encore très claires, l'une concernant le choix de la langue étrangère, l'autre concernant l'âge du démarrage.

S'agissant du choix de la langue, on est dans la conjugaison de l'ambiguïté et de l'hypocrisie ; les contorsions de langage et les contradictions sont la règle. Officiellement, le choix est très large, concrètement, l'hégémonie de l'anglais est absolue ; on affirme haut et fort (institution scolaire et parents) que la diversité linguistique et culturelle est une nécessité incontournable, mais on ne choisit que l'anglais ou presque ... (le rapport Thélot qui préparait la réforme Fillon en 2004 avait le mérite de proposer franchement « l'anglais de communication internationale " (?) comme langue obligatoire à l'école élémentaire à partir du CE2, mais ce fut refusé en haut lieu ... et on a préféré laisser en l'état).

8 L'anglo-américain est la langue du plus fort, militairement et économiquement, il faut donc apprendre cette langue ; il est exact que la demande sociale dominante est d'apprendre l'anglais mais la conséquence évidente, c'est qu'à moyens constants, l'offre des autres langues ne peut alors que diminuer : cercle vicieux par excellence qui souligne les contradictions entre les déclarations d'intentions qui prônent la diversité linguistique et la réalité institutionnelle qui sur - privilégie une seule langue.

On s'étonnera tout de même de constater que devant cette situation, les autorités ministérielles ne proposent pas de solutions qui permettraient de respecter les minorités et d'être ainsi en accord avec leurs grandes déclarations prônant la diversité linguistico - 
culturelle, sans le moins du monde d'ailleurs empêcher l'apprentissage généralisé de l'anglais. Par exemple, une solution est de commencer obligatoirement à l'école élémentaire par apprendre une autre langue que l'anglais, (langue étrangère et / ou langue régionale), et de ne démarrer l'anglais par conséquent qu'au niveau du collège. Mais bien d'autres stratégies et chronologies d'apprentissage des langues existent, et le rôle de l'éducation nationale d'un pays n'est-il pas d'éduquer le public, d'expliquer des choix respectueux de tous, et finalement de formuler des programmes et des instructions conformes aux valeurs affichées, et singulièrement celle de la diversité lorsqu'il s'agit de langues?

10 S'agissant de l'âge du démarrage des langues étrangères, c'est-à-dire de la thématique de l'apprentissage précoce, il faut dire d'abord qu'on a quelquefois raconté bien des sottises concernant les vertus mythiques d'un apprentissage le plus précoce possible.

11 Sans entrer vraiment ici dans cette problématique qui mériterait de longs développements, disons qu'on a beaucoup fantasmé sur l'enfant qui « s'imbibe de langue étrangère comme une éponge ", sur les miracles de "l'immersion ", sur la facilité et l'efficacité de tels apprentissages dès l'école maternelle. Il faut être conscient que tout dépend, à l'école, des conditions environnementales (l'exposition à deux langues en famille est un autre débat). Cela dépend bien sûr de l'état d'évolution de la fonction langage du jeune enfant, des relations que le locuteur entretient avec lui, de la qualité professionnelle de l'enseignant, du temps d'exposition journalier à la langue étrangère, des sujets abordés etc. etc.

S'il est exact qu'au niveau de l'oreille et de la phonation un « plus » est souvent souligné (meilleur « accent ») en faveur de l'enseignement précoce, il est bien difficile de déclarer que " plus on commence tôt mieux ça vaut pour maîtriser une langue étrangère »; de telle façon qu'il est probablement illusoire de penser que l'on connaîtra mieux l'anglais en l'apprenant dès la maternelle (d'autant que l'on peut craindre le pire, quelquefois, avec un enseignement qui dure pendant plus de dix ans consécutifs : rabâchages, lassitude, ennui...).

Les multiples évaluations qui ont été faites depuis le lancement de l'EPLV montrent clairement qu'il n'est pas possible de pointer une amélioration significative de la maîtrise d'une langue étrangère en commençant dès le début de l'école élémentaire, les professeurs de 6e (re)commençant d'ailleurs le plus souvent à zéro, ignorant ou minimisant les acquis ...

14 Il semble bien finalement qu'il soit nécessaire de reconsidérer l'ensemble de la problématique de l'enseignement des langues à l'école primaire, en relation avec une analyse politique sérieuse de l'état et de l'évolution des relations humaines dans le monde, en relation aussi avec les apports et les éclairages de chercheurs nationaux et étrangers aussi divers que sociologues et sociolinguistes, économistes et anthropologues, didacticiens et psychologues, en relation enfin avec les praticiens et les pédagogues. 
Il s'agit d'abord de recentrer le débat en explicitant parfaitement les finalités et objectifs attendus et sans viser uniquement le très court terme ... car il est clair que les enfants de 5 ans d'aujourd'hui pourront vivre encore bien sûr dans les années 2080 ...

\section{Un enseignement des langues à l'école élémentaire qui vise d'abord « l'éducation linguistique » de l'élève}

La première question à se poser est bien en effet de se demander (et la réponse ne va pas de soi) pourquoi il est important d'enseigner plusieurs langues à l'école élémentaire?

Est-ce parce que c'est l'âge le plus favorable ? Pour faire comme nos voisins ? Pour « prendre de l'avance » dans l'apprentissage de l'anglais?

Les réponses institutionnelles ne sont pas claires ; elles pourraient l'être pourtant, compte tenu de cette idée centrale exprimée depuis toujours dans les textes officiels, selon laquelle l'école élémentaire a pour fonction essentielle de développer une éducation équilibrée et épanouissante dans tous les domaines de la vie sociale. Dans le préambule des programmes de 2002 pour l'école élémentaire (et comme toujours dans ce type de texte depuis plus d'un siècle) on parle de tout mettre en oeuvre pour développer l'éducation physique, l'éducation civique, l'éducation artistique, l'éducation musicale, l'éducation scientifique et technique ... mais on ne parle pas de développer une " éducation linguistique ». On insiste bien sûr sur l'importance de la maitrise de la langue française, mais cette maitrise n'est-elle pas un cas particulier de "l'éducation linguistique»? On est là au coeur d'une réflexion qui vaut la peine d'être approfondie et qui, en tous cas, devrait permettre de situer et de donner du sens et des objectifs à l'enseignement et l'apprentissage d'autres langues que le français à l'école élémentaire.

19 Apprendre la langue française en apprenant aussi et parallèlement d'autres langues (selon naturellement des posologies et des rythmes différents) ne serait-il pas un bon moyen d'apprendre mieux cette langue française, tout en développant cette « éducation linguistique » qui permettra d'apprendre mieux et plus vite, tout au long de la vie, d'autres langues?

Cette perspective d'" éducation linguistique ", positionnant les langues régionales et étrangères comme faisant partie du même champ disciplinaire que la langue maternelle n'est pas nouvelle (Titone, universitaire italien, a développé cette idée voici une trentaine d'années) et il est bien clair que sa mise en oeuvre ne va pas de soi. Mais n'est-ce pas un concept à approfondir, un objectif à viser, répondant tout à la fois à la mondialisation des communications et au respect de la diversité linguistique et culturelle?

21 Le temps manque ici pour montrer comment, dans les dispositifs d'enseignement bilingue (avec langue régionale ou langue étrangère associée à la langue française) on a pu observer et analyser l'émergence de compétences métalinguistiques transversales améliorant l'apprentissage du français, comment par conséquent cette " éducation linguistique » facilite l'apprentissage de nouvelles langues.

22 Tentons seulement maintenant, si l'on admet cette idée de considérer l'apprentissage d'une langue autre que la langue française à l'école primaire comme ayant pour fonction essentielle de développer une "éducation linguistique ", d'en esquisser les conséquences au niveau des pratiques scolaires. 


\section{Quelques grands axes méthodologiques et didactiques} première ... Evlang.

D'abord, ne pas hésiter à relier la langue nouvelle à la langue nationale (langue officielle, souvent maternelle).

Il s'agit de bien faire intérioriser que la langue française est un cas particulier des langues du monde (même si elle est naturellement à privilégier, cultiver et choyer dans son pays), mais il s'agit de la même matière "langue ». Cela veut dire que l'on devra mettre en relation cette nouvelle langue avec la langue française, développer et exploiter pédagogiquement des comportements intellectuels naturels des apprenants comme par exemple ceux de la découverte, de la surprise, du jeu et aussi de la comparaison. Lorsqu'il s'agit de langues proches, ces analyses comparatives sauvages et intuitives pourront être judicieusement exploitées pour le plus grand profit de l'apprentissage de la langue française et des langues concernées. Le comparatif / contrastif, l'intercompréhension entre langues de la même famille (explorées dans des programmes européens tels que Galatea, Eurom4, Eurocom..), l'éveil aux langues (l'« awareness » et Evlang, autre programme européen), l'approche par des langues régionales proches du français (catalan, occitan, corse ...) ou non (breton, alsacien, basque ...) toutes ces pistes sont utiles pour développer « l'éducation linguistique ».

Ensuite, donner du sens à ces nouvelles langues en faisant quelque chose avec, en s'en servant comme outil pour apprendre ... Dans le domaine des langues, « entreprendre pour apprendre » est beaucoup plus vrai que la fameuse devise « apprendre pour entreprendre "; on peut faire des activités physiques avec la nouvelle langue, des activités artistiques , mais aussi des activités à coût cognitif réputé plus élevé comme les mathématiques (voir les classes bilingues français - basque ou français - breton où les maths sont enseignées largement en langue régionale, avec succès).

Pratiquer l'écrit en même temps que l'oral, à partir du cours préparatoire ; des observations et évaluations dans les écoles françaises à l'étranger où deux langues coexistent de fait, montrent qu'on apprend mieux à lire avec deux langues, que les comportements de lecteur (recherches d'hypothèses, anticipations pour faire du sens, rapidité et efficacité de lecture) sont améliorés lorsqu'une autre langue existe à côté de la

Commencer assez tôt, sans doute, mais pas avant que la fonction langage soit bien en place, c'est-à-dire pas avant l'âge de 4 à 5 ans ; (notons que ceci n'est valable que lorsque la nouvelle langue n'existe pas en famille ; si au contraire cette dernière est présente dans l'environnement familial, on est dans un autre cas de figure).

Entrer par la culture en même temps que par le volet linguistique ; on sait bien que la langue est un produit culturel, historiquement comme pédagogiquement.

Utiliser largement les outils didactiques que le Conseil de l'Europe a élaborés, comme peuvent être les différents portfolios, mais aussi les programmes européens de Bruxelles, comme par exemple, s'agissant de l'école élémentaire, les conclusions du programme

On pourra aussi naturellement s'inspirer des expériences des pays voisins (Espagne, pays du nord de l'Europe ...). 
31 Et puis bien sûr, il s'agira de développer la formation des maîtres ; le chantier est là largement ouvert ; certes des initiatives individuelles existent, et l'on a beaucoup à apprendre à ce propos dans les pratiques quotidiennes des maîtres qui enseignent dans les sections bilingues « langue française - langue régionale ». Mais les IUFM (à quelques exceptions près, comme en Alsace) tardent à inscrire significativement dans leurs programmes des modules pour apprendre aux maîtres à introduire un enseignement de langue autre que le français à l'école élémentaire.

32 La recherche didactique en ce domaine n'est pas non plus très florissante, mais elle existe et se développe ici ou là en France, en Suisse, en Belgique, au Canada.

\section{Finalement, que conclure?}

33 L'enseignement des langues autres que le français existe à l'école élémentaire, se développe et cette évolution semble irréversible.

34 L'objectif fondamental de cet enseignement devrait sans doute être redéfini dans le sens d'un développement prioritaire d'une « éducation linguistique » de base, aussi utile à la langue première qu'aux autres langues.

Certains lieux sont probablement plus avancés que d'autres en France au plan de la mise en oeuvre pédagogique, et notamment les lieux où existent des enseignements de langues régionales associés à l'enseignement en français.

La formation des maîtres est urgente ; on peut se réjouir que dorénavant le concours de recrutement de ces maîtres comporte une épreuve de langues.

Mais il reste beaucoup à faire et il faut être conscient qu'en France, pour des raisons à dominante historique, les évolutions en ce domaine vont être quelquefois conflictuelles, elles sont difficiles et prendront par conséquent beaucoup de temps.

\section{RÉSUMÉS}

Pour diverses raisons, l'enseignement de langues étrangères à l'école primaire française se met en place lentement et bien difficilement ; l'essentiel serait sans doute de mettre en relation l'apprentissage de ces langues étrangères avec celui de la langue officielle, afin de contribuer à construire une éducation au plurilinguisme indispensable dans le monde contemporain ; pour y parvenir, il conviendrait de mettre en oeuvre de nouvelles méthodologies.

For various reasons, the introduction of foreign languages at primary school level is still slow and rather difficult in France. It would be important to articulate their teaching with that of the official (national) language, so as to develop a kind of plurilingual education, much needed in today's world. To reach this aim, new approaches should be developed.

\section{AUTEUR}

\section{JEAN DUVERGER}

Inspecteur de l'éducation honoraire, Président de l'Association pour le développement de l'enseignement bi / plurilingue 